\title{
Vaginal flora and urinary and vaginal group B streptococci in early pregnancy
}

\author{
Linnéa Ekström', Annsofie Adolfsson ${ }^{2,3}$, Henrik Ericson4, Georgios Poutakidis², Georgios Charonis ${ }^{5,6}$ and Per-Göran Larsson ${ }^{2 *}$ \\ *Correspondence: p-g.larsson@vgregion.se \\ 'Karolinska Institute, Stockholm, Sweden. \\ 2Department of Obstetrics and Gynaecology, Skaraborg Hospital, Skövde, Sweden. \\ ${ }^{3}$ School of Health and Medical Sciences, University of Örebro, Örebro, Sweden. \\ ${ }^{4}$ School of Life Sciences, University of Skövde, Skövde, Sweden. \\ ${ }^{5}$ Department of Obstetrics and Gynaecology, University of Linköping, Linköping, Sweden. \\ ${ }^{6}$ Mitera General and Maternity Hospital, Athens, Greece.
}

\begin{abstract}
Background: Bacterial vaginosis (BV) is a risk factor for premature birth and group B streptococci (GBS) colonizing the vagina are etiological agents of neonatal infections. Significant growth of GBS in the vagina has been assumed to be detectable through urinary culture. The aim was to investigate the correlation between BV and the presence of GBS in qualitative vaginal or quantitative urinary culture, since this could predict a higher risk for perinatal morbidity.

Design and setting: A consecutive prospective study of women in early pregnancy included 3101 women between 2007 and 2010 , in a region of south-western Sweden.

Methods: Vaginal and urine samples were obtained from women in early pregnancy at maternity health care clinics. BV was diagnosed according to the Ison/Hay classification. GBS in urine were detected in amounts as low as $100 \mathrm{CFU} / \mathrm{ml}$. Vaginal culturing for GBS was obtained from a selected group of 481 women.

Results: There was no difference in the prevalence of GBS in the urine among women with BV compared with women with lactobacilli flora (OR 0.7; 95\% CI 0.4-1.1). Vaginal presence of GBS was found among $17.3 \%$ of women with BV and among $23.5 \%$ of women with lactobacilli flora (OR 0.7; 95\% CI 0.3-1.4). Among the 105 women who had vaginal GBS, the urine culture of GBS was positive in only $21.9 \%$ of cases.

Conclusions: Even though women with BV. have much higher concentration of bacteria in the vagina, they do not necessarily have more GBS in the vagina or urine. The modest correlation between positive vaginal culture and positive urine culture of GBS question the value of urinary culture for detection of vaginal GBS.
\end{abstract}

Keywords: Bacterial vaginosis, group B streptococci, pregnancy, vagina

\section{Introduction}

Bacterial vaginosis is a vaginal disease that causes a malodorous discharge, but it can also be asymptomatic. Nevertheless, it is one of the most common causes of vaginitis and reasons for women to seek medical help [1]. BV is a change in the vaginal flora, both in number and type of bacteria. Instead of the ordinary lactobacilli; anaerobic bacteria together with Gardnerella vaginalis and Mycoplasma hominis colonizes the vagina and in higher amount than the lactobacilli. The concentration of vaginal bacteria is more than 1000 times higher in the vagina among women with BV compared with women with lactobacilli flora; The bacterial count increases from $10^{5}-10^{6}$ to a least $10^{8}-10^{9} \mathrm{CFU} / \mathrm{ml}$ with Gardnerella vaginalis as the dominating species. The counts of nearly all other bacteria are also raised. For pregnant women, BV doubles the risk of preterm delivery, that is delivery before gestation week 37+0 [2].

Group B streptococci are gram positive bacteria present in aerobic and anaerobic environments. It colonizes the gastrointestinal and genitourinary tract of men and women and can be transmitted through sexual contact. The bacteria can also be transmitted from a pregnant woman to her child during childbirth [3,4]. Since the 1970 s GBS has been the most common infectious cause of early neonatal morbidity [4]. Through the treatment of pregnant women with prophylactic intrapartum antibiotics, the number of newborns with earlyonset disease caused by GBS has decreased by approximately $70 \%$ [3]. Vaginal culture for GBS is therefore routinely performed during pregnancy, but in Scandinavia, antibiotic prophylaxis is undertaken according to risk factors for early-onset group $B$ streptococcal disease. The risk factors includes a GBS-positive urine culture [5], since it is proposed that a GBS positive urine culture indicates a high concentration of GBS in the vagina. Vaginal culture methods reveal only the presence or absence of GBS; therefore urine culture methods must be employed in order to acquire quantitative measurements of the degree of colonization.

Hypothetically, women with both BV and vaginal GBS could have a high concentration in the vagina of not only typical 
Ekstrom et al. Gynecology 2013,

http://www.hoajonline.com/journals/pdf/2052-6210-1-6.pdf

doi: $10.7243 / 2052-6210-1-6$

BV bacteria such as G. vaginalis, Peptostreptococcus spp., Prevotella spp. and Atropobium vaginae, but also have a high concentration of GBS in the vagina. A high count of vaginal GBS consequently increases the risk of the bacteria being transmitted to the child during delivery. Accordingly, a positive culture of urinary GBS could indicate heavy GBS colonization of the vagina. A correlation between high levels of GBS in the urine and $B V$ could therefore be an indication of having both vaginal $G B S$ and $B V$, which would be a strong risk factor for perinatal morbidity. BV increases the risk of preterm birth [2], and the mortality rate of early-onset disease is higher among children born preterm than among children born after 37 gestational weeks [6].

The aims of this study were to investigate the prevalence of BV during early pregnancy and to analyze the correlation between $B V$ and GBS, both in qualitative vaginal culture and in quantitative urinary culture of GBS. The aim was also to compare urine culture of GBS with vaginal culture of GBS.

\section{Material and methods}

A consecutive prospective study of bacterial vaginosis during early pregnancy began in 2007 in the Skaraborg region of Västra Götaland. Not all of the fifteen maternity health care clinics were involved from the start of this study, but they all came to be included as the study progressed. Inclusion criteria were all pregnant women between 2007 and 2010. No women were excluded because of prior antibiotic use.

Approximately 5000 sample kits were distributed to maternity health care clinics. Women were included on their first visit to a maternity health care clinic, usually between gestation weeks 10-12, where a vaginal sample and a vaginal $\mathrm{pH}$ test were either self-administered or completed by midwives. The vaginal sample was transferred to a microscope slide, air dried, and transported to the gynecological clinic in Skövde where it was microscopically examined using a phase contrast microscope at 400x magnification, according to the Ison/Hay criteria for the diagnosis of BV [7]: The Ison/ Hay criteria have been developed using Gram-stained smears with oil immersion at 1000x magnification but since Ison/ Hay criteria assess the type of flora and do not indicate a count of individual bacteria, which is done using Nugent's criteria, it is possible to use these criteria with non-stained smears at less magnification [8]. The vaginal flora is divided into the following three different categories: normal (grade 1), intermediate (grade 2), and BV (grade 3), depending on the relative amount of lactobacillus morphotypes as compared to the Gardnerella morphotypes. BV was defined as grade 3 according to Ison/Hay. In all vaginal samples at least four fields were evaluated, and the estimated number of Lactobacillus morphotypes and Gardnerella morphotypes, together with the estimated number of curved rods (Mobiluncus morphotype) per high power field were noted as intervals on an ordinal scale (range $0-100000$ bacteria per field). Estimation of numbers of bacteria, in intervals, was performed assuming that the number of bacteria (1-30) noted in a part of a representative microscopic field can be used to estimate the approximate number of bacteria in the whole field [9]. We deliberately chose not to use the Nugent classification as this has numerous disadvantages compared with the Ison/Hay method $[7,8]$. All patients with BV were treated with vaginal clindamycin cream and a further follow-up vaginal sample after 10 weeks, data not shown.

During the first visit, a urine sample for culture was also taken. The etiological bacteria was known and reported from the microbiological institution if bacteriuria included $\geq 100000$ $\mathrm{CFU} / \mathrm{ml}$. In contrast, if GBS growth was noticed, the amount was confirmed, even if it was lower than $100000 \mathrm{CFU} / \mathrm{ml}$. Culture for GBS in urine was performed on a plate constructed of two halves, each with different culture substances; Blood agar and Chrome agar. $10 \mu \mathrm{L}$ of urine was extracted to each half of the plate. The plate was incubated aerobically for 24 hours at $36^{\circ} \mathrm{C}$ and thereafter read manually regarding the colour and shape of the bacterial colonies. If the bacterial growth was similar to the one of GBS, further culture was done to distinguish GBS from Enterococcus. This was made with culture on two plates; Esculin agar (bacterial control was E. faecium) and the CAMP-test (the Christie, Atkins and Munch-Petersen) with $\beta$-toxin producing S. Aureus (bacterial control was CAMPpositive GBS). Both plates were incubated aerobically for approximately 15 hours at $36^{\circ} \mathrm{C}$. The Enterococcus hydrolysis Esculin to Esculetin and a reaction on this plate is therefore positive for growth of Enterococcus. The CAMP-test shows growth of GBS through the mechanism of which GBS enhances the betatoxin produced by S. Aureus, and is shown through a reaction caused by haemolysis.

Over a period of two months, vaginal culture was also performed in two maternity health care clinics to investigate the frequency of vaginal GBS. These samples were taken at the same time as the vaginal samples. Each sample was taken with a dry Copan vaginal swab (114C.USE; regular single plastic applicator) from the lower part of the vagina. The samples were then transported in Amies agar transport gel with charcoal to the Skaraborg hospital laboratory.

Vaginal swabs were incubated in selective enrichment media, Todd Hewitt broth with antibiotics (bioMérieux $A B$, prod\# 42116), for 24 hours at $37^{\circ} \mathrm{C}$. Samples were then inoculated onto chromID ${ }^{\mathrm{TM}}$ Strepto $B$ agar (bioMérieux $A B$, prod\# 43461) according to the manufacturer's instructions and GBS colonies were verified by Slidex ${ }^{\circledR}$ Strepto Plus B (bioMérieux AB, prod\# 58819) [10].

All demographic data such as age and parity were collected from the computer-based Obstetrix medical register. Pregnancy outcomes are to be presented in another publication.

The data were transferred to the Statistical Package for the Social Sciences (SPSS, version 19.0). The statistical methods used were a t-test, an odds ratio (OR), and a bivariate analysis with a Spearman's rank correlation coefficient. A 95\% confidence interval ( $\mathrm{Cl}$ ) was used. P-values $\leq 0.05$ were considered significant. 


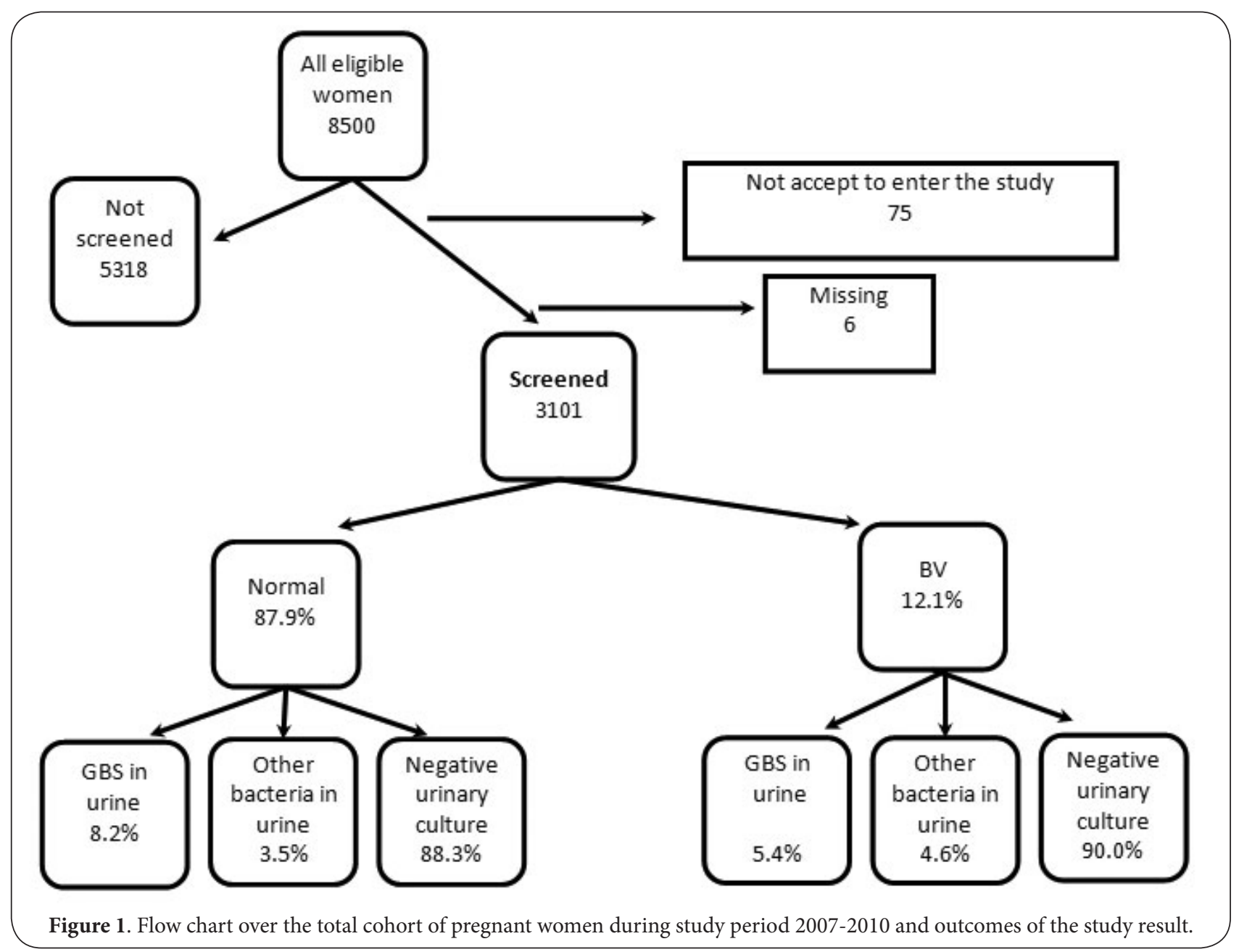

The participants were informed verbally and through a written and signed consent form. Ethical approval was received from the regional ethical review board (EPN) in Gothenburg (2006): number 443-06.

\section{Results}

A total of 3182 samples were retrieved by the gynaecological clinic in Skövde, and 3107 women agreed to be included in the study. The vaginal specimen was not possible to specify among six women. The data from these women were excluded, leaving 3101 women's vaginal samples to be classified according to Ison/Hay [7]. The number of eligible women who delivered during the period was around 8500; thus, the total number of women investigated represented around $36 \%$ of the total possible sample (3101/8500) (Figure 1). This is because not all maternity health care clinics were involved from the beginning of the study, but rather they were integrated as the study progressed. The number of women included increased from 260 to 930 per year during the study period. Urine culture of GBS was performed with 2983 women, and vaginal culture of GBS was performed for only for a selective number of 481 women taken consecutively from two maternity health care clinics during two months.

The prevalence of BV was 12.1\% (375/3101). Among BV positive women, the prevalence of GBS in the urine was lower than among women with normal vaginal flora but not significantly (OR 0.7; 95\% Cl 0.4-1.1) (Table 1).

Women with BV were slightly younger than women with normal vaginal flora; with the average ages being 28.2 and 30.2 respectively and were more often nulliparous (OR 1.7; 95\% Cl 1.3-2.2) (Table 1).

The quantitative analysis of GBS in urine indicated that there was no significant difference in the amount of GBS in urine between women with BV or normal vaginal flora $(p=0.7)$ (Table 2). The mean values of quantitative culture of GBS in urine were on average $21000 \mathrm{CFU} / \mathrm{ml}$ in women with BV and $24000 \mathrm{CFU} / \mathrm{ml}$ in women with normal vaginal flora.

As mentioned earlier, both number and type of vaginal bacteria is different in women with or without BV. Among women with BV, the mean number of the dominant bacteria with Gardnerella morphotypes/visual field was 8037, compared with women with normal vaginal flora who had a mean number 
Ekstrom et al. Gynecology 2013,

http://www.hoajonline.com/journals/pdf/2052-6210-1-6.pdf

doi: $10.7243 / 2052-6210-1-6$

Table 1. Background data of women screened for BV during early pregnancy and the presence of positive urine cultures.

\begin{tabular}{lccc}
\hline & BV positive & Normal flora & Significant \\
\hline $\begin{array}{l}\text { Mean age (year) } \\
\text { Nullipara (\%) }\end{array}$ & 28.5 & 30.2 & $\mathrm{p}<0.05$ \\
\hline & 58.8 & 41.2 & $\mathrm{p}<0.05$ \\
\hline $\begin{array}{l}\text { GBS in urine (\%) } \\
>100 \mathrm{CFU} / \mathrm{ml}\end{array}$ & 5.4 & 8.2 & 0.64 (CI 0.40-1.04) \\
$\begin{array}{l}\text { GBS in urine (\%) } \\
>100000 \mathrm{CFU} / \mathrm{ml}\end{array}$ & 0.9 & 1.7 & 0.51 (CI 0.16-1.62) \\
$\begin{array}{l}\text { Other bacteria (\%) } \\
>100000 \mathrm{CFU} / \mathrm{ml}\end{array}$ & 4.6 & 3.5 & $1.3(\mathrm{CI} 0.80-2.2)$ \\
\hline
\end{tabular}

Table 2. The amount of GBS in urine among women with BV or lactobacilli.

\begin{tabular}{ccccc}
\hline & $\begin{array}{c}\text { BV and GBS } \\
\text { in urine }\end{array}$ & $\%$ & $\begin{array}{c}\text { lactobacilli and } \\
\text { GBS in urine }\end{array}$ & $\%$ \\
\hline all & 19 & $5.7 \%$ & 215 & $8.5 \%$ \\
$\geq 100000 \mathrm{CFU} / \mathrm{ml}$ & 3 & $0.9 \%$ & 44 & $1.7 \%$ \\
$\geq 5000 \mathrm{CFU} / \mathrm{ml}$ & 5 & $1.5 \%$ & 76 & $3.0 \%$ \\
$>1000 \mathrm{CFU} / \mathrm{ml}$ & 7 & $2.1 \%$ & 55 & $2.2 \%$ \\
$\leq 1000 \mathrm{CFU} / \mathrm{ml}$ & 4 & $1.2 \%$ & 40 & $1.6 \%$ \\
\hline
\end{tabular}

Table 3. Comparison of qualitative vaginal and quantitative urinary culture of GBS.

\begin{tabular}{ccccc}
\hline & \multicolumn{3}{c}{ Vaginal GBS } \\
\hline \multirow{2}{*}{ Urinary GBS } & Positive & Negative & Total \\
& & 23 & 7 & \multirow{2}{*}{30} \\
& \multirow{2}{*}{ Negative } & $20.7 \%$ & $2.0 \%$ & \\
& & 88 & 353 & 441 \\
& \multirow{2}{*}{ Total } & 111 & $36.0 \%$ & \\
& & $100 \%$ & $100 \%$ & 471 \\
\hline
\end{tabular}

${ }^{a}$ Bacteria $\geq 100 \mathrm{CFU} / \mathrm{ml}$

of 426 lactobacillus morphotypes/visual field. There was no correlation between the amount of GBS/ml urine and the number of vaginal lactobacilli morphotypes or the number of Gardnerella morphotypes per visual field (representing BV) (Spearman correlation coefficient $\rho=0.057, p=0.34$ ).

Qualitative vaginal culture of GBS where we also had urinary culture was obtained from 470 women; GBS was present in $23.6 \%(111 / 470)$ of the cases. However, only 23 cases (20.7\%) had both GBS in the vagina and urine (Table 3). The urinary culture had a sensitivity of 0.22 and a specificity of 0.98 in identifying the presence of vaginal GBS. BV positive women had vaginal GBS in $17.3 \%$ (9/52) of the cases, while women with normal vaginal flora had vaginal GBS in $23.5 \%$ of the cases (96/408) (OR 0.7; 95\% Cl 0.3-1.4).

Bacteria in urine, other than GBS, were included if the amount of bacteria was $\geq 100000 \mathrm{CFU} / \mathrm{ml}$, in accordance with the definition of UTI. Three bacterial species dominated: E. coli $59.6 \%$ (65//109), Enterococcus 10.1\% (11/109), and Coagulase Negative Staphylococci 11.1\% (12/109), representing 80.7\% of the 13 different bacteria found in total. Bacteriuria with bacteria other than GBS was not significantly higher among BV-positive women (4.8\%) than in women with lactobacilli (3.6\%) (OR 1.3; Cl: 0.8-2.3).

\section{Discussion}

The results indicate that GBS in urine (8.2\%) or vagina $(24.6 \%)$ is less common among women with BV $(5.4 \%$ and $16.7 \%$, respectively) and that the amount of GBS in urine has no correlation with BV. Our hypothesis that BV promotes a high number of GBS in the vagina, which further leads to a high number of GBS in the urine, was therefore rejected.

Our finding of the high frequency of vaginal GBS colonization among women with negative urinary culture questions the value of urinary culture for the detection of GBS in the vagina, which is used in some parts in Sweden. A negative urinary culture for GBS can not exclude women for prophylactic antibiotics during labour.

A study of the correlation between BV and quantitative culture of GBS in urine has not been published before. Since $\mathrm{BV}$ is a risk factor for prematurity [2] and GBS is a cause of neonatal morbidity [6], this study of the correlation between BV and GBS is important. To investigate the correlation between BV and GBS, both qualitative and quantitative measures of GBS were used. Normally, colonization rates of GBS are presented using vaginal culture, a qualitative method by which it is only possible to verify the presence of GBS, not how heavy the colonization are. Since one aim of our study was to investigate the correlation between BV and the amount of GBS, urinary culture was necessary. This because quantitative culture of vaginal samplings is difficult because of the imprecision in extracting the exact amount of discharge needed for comparable culture. All urine samplings were taken during the first trimester. As other authors have noted, having GBS at one point indicated a continuous colonization of GBS even if the GBS disappears after treatment. Therefore we have also analysed the positive urine cultures which were made later on in the pregnancy. These showed that 62 women had a positive urine culture for GBS later on in pregnancy. These cultures were made on average 13 weeks later. Including the data from these women in the analysis did not change the results as the relationship between $\mathrm{BV}$ and lactobacilli was the same. However, positive urine cultures of bacteria other than GBS were found in 26 cases in later stages of pregnancy. If these cultures are included in the analysis there is a significant RR of 1.7 (1.2-2.4) to having a positive urine culture after having BV. Since all patients were treated with clindamycin, this could be an effect of the treatment rather than BV itself.

Compared with earlier studies, the prevalence of $B V$ and GBS was as expected; Håkansson et al., reported a vaginal/ 
Ekstrom et al. Gynecology 2013,

rectal GBS colonization rate of $25.4 \%$, a rate similar to that in our results (23.7\%) [11]. We did not however perform rectal culture for GBS as we have little knowledge as to whether or not rectal bacteria could infect the vagina. However, transmission to newborns and neonatal morbidity was not investigated in this study.

The limitations of the study are that only 470 women had a vaginal culture. We think however that because these women were selected from two maternity health care centres we should have a representative sample of vaginal cultures.

The absence of correlations between GBS and BV could be due to BV inhibiting the growth of vaginal GBS. This could also imply that urinary culture for GBS does not accurately reflect the state of vaginal colonization by GBS. Consequently, this could contradict the use of urinary culture as an indicator of vaginal colonization by GBS. Since only about $21 \%$ of women with GBS-positive vaginal culture had positive urine culture, the value of negative urinary culture among pregnant women is therefore questioned. Even among women with lactobacilli, no correlation with the amount of GBS in the urine was found, which may suggest that urinary GBS is not necessarily a sign of heavy colonisation of GBS in the vagina. This challenges the use of urinary GBS culture to be used as an indicator for vaginal GBS, since it could exclude women from being given intra-partum antibiotic prophylaxis (IAP).

\section{Competing interests}

The authors declare that they have no competing interests.

\section{Authors' contributions}

A Adolfsson, G Charonis, $\mathrm{H}$ Ericson and PG Larsson planed and stared the study. L Ekström, G Poutakidis PG Larsson contributed with data collection. All authors contributed to the manuscript writing.

\section{Acknowledgement and funding}

We are grateful to the participants and staff of the maternity healthcare clinics in Skaraborg. The study was supported by grants from the research and development unit in Skaraborg and at Skaraborg hospital.

\section{Publication history}

EIC: Christos lavazzo, Royal Surrey County Hospital, UK. Received: 14-Apr-2013 Revised: 26-Jun-2013

Accepted: 02-Jul-2013 Published: 18-Jul-2013

\section{References}

1. Verstraelen $H$, Verhelst $R$, Vaneechoutte $M$ and Temmerman $M$ : The epidemiology of bacterial vaginosis in relation to sexual behaviour. BMC Infect Dis 2010, 10:81. | Article | PubMed Abstract | PubMed Full $\underline{\text { Text }}$

2. Leitich $H$, Bodner-Adler B, Brunbauer $M$, Kaider $A$, Egarter $C$ and Husslein $\mathrm{P}$ : Bacterial vaginosis as a risk factor for preterm delivery: a metaanalysis. Am J Obstet Gynecol 2003, 189:139-47. | Article | PubMed

3. Nandyal RR: Update on group $B$ streptococcal infections: perinatal and neonatal periods. J Perinat Neonatal Nurs 2008, 22:230-7. | Article | PubMed

4. Verani JR, McGee $L$ and Schrag SJ: Prevention of perinatal group B streptococcal disease--revised guidelines from CDC, 2010. MMWR Recomm Rep 2010, 59:1-36. | Article | PubMed
5. Jacobsson B, Håkansson S. Infpreg: Grupp B streptokocker [Group B streptococci] (in Swedish). I Website

6. Hakansson $S$ and Kallen K: Impact and risk factors for early-onset group B streptococcal morbidity: analysis of a national, populationbased cohort in Sweden 1997-2001. BJOG 2006, 113:1452-8. | Article | PubMed

7. Ison CA and Hay PE: Validation of a simplified grading of Gram stained vaginal smears for use in genitourinary medicine clinics. Sex Transm Infect 2002, 78:413-5. | Article | PubMed Abstract | PubMed Full Text

8. Larsson PG, Carlsson B, Fahraeus L, Jakobsson T and Forsum U: Diagnosis of bacterial vaginosis: need for validation of microscopic image area used for scoring bacterial morphotypes. Sex Transm Infect 2004, 80:637. | Article | PubMed Abstract | PubMed Full Text

9. Koch AL. Growth measurent. In: Gerhardt P, Murray RGE, Wood WA, Krieg NR, eds. Methods for general and molecular bacteriology. Washington DC: American Society for Microbiology 1994, 248-77.

10. Claesson B. Metodbeskrivning klinisk mikrobiologi. [Method description clinical microbiology] (in Swedish. No abstract available) Unilabs 2010, 1-10:11.

11. Hakansson $S$, Axemo P, Bremme K, Bryngelsson AL, Wallin MC, Ekstrom CM, Granlund M, Jacobsson B, Kallen K, Spetz E and Tessin I: Group B streptococcal carriage in Sweden: a national study on risk factors for mother and infant colonisation. Acta Obstet Gynecol Scand 2008, 87:508. | Article | PubMed

\section{Citation:}

Ekström L, Adolfsson A, Ericson H, Poutakidis G, Charonis $\mathrm{G}$ and Larsson P-G. Vaginal flora and urinary and vaginal group $B$ streptococci in early pregnancy. Gynecol. 2013; 1:6. http://dx.doi.org/10.7243/2052-6210-1-6 